\title{
A T-branch diplexer based on directional couplers and resonant cavities in photonic crystal
}

\author{
Amel Labbani ${ }^{1, *}$, Imene Moumeni ${ }^{1}$, and Faiza Bounaas ${ }^{1}$ \\ ${ }^{1}$ Laboratory of Hyperfrequency and Semiconductors (L.H.S), Department of Electronics, Faculty of Technology and Sciences, \\ University of Mentouri brothers Constantine 1, Constantine 25000, Algeria
}

\begin{abstract}
In this paper a T-branch optical diplexer in two dimensional (2D) photonic crystal (PhC) to select two telecommunication wavelengths $1493.6 \mathrm{~nm}$ and $1553 \mathrm{~nm}$ is investigated .In our design directional couplers (DC) and resonant cavity (RC) are utilized. A square lattice of silicon (Si) rods in air is used as fundamental structure. The coupling regions consist of three entire rows of decreased Si rods. Plane wave expansion method (PWE) and finite difference time domain (FDTD) method are utilized to analyze and simulate the characteristics of the designed device. The average transmission efficiency of our proposed diplexer is about $99.75 \%$. High quality factor and extremely small crosstalk were achieved. The total size of the suggested design is $272.214 \mu \mathrm{m}^{2}$, which is very suitable for nanotechnology based demultiplexing applications.
\end{abstract}

\section{Introduction}

Recently, many attempts have been focused on developing micro and nano photonic devices by using Photonic Crystals (PCs) due to their unique properties such as ultrasmall size, compactness, and massmanufacturing possibilities. PhCs are optical materials with a periodic dielectric constant on one, two or three directions in space $[1,2]$ This periodic variation is responsible for the formation of regions of prohibited frequencies known as photonic band gaps (PBGs) [3]. By creating different defects in $\mathrm{PhCs}$, allowed modes appear within PBGs. This will give various applications using the PBG concept, such as photonic crystal waveguides [4] switch [5], etc. Several topologies have been proposed for designing demultiplexers based $\mathrm{PhCs}$ using coupler [6] waveguide couplers [7,8] line defect [9] coupled cavity waveguides [10-12] ring resonator [13-15] heterostructure [16] core-shell rods defects [17], etc. Recently many works have been devoted for designing $\mathrm{T}$ shaped two channel in two dimensional photonic crystal demultiplexer are proposed [18] It is known that $\mathrm{T}$-junction structures have poor transmission due to the bending loss and absence of conformity in the joints. The optical wave gets scattered at the bends and most of the incoming signal is reflected. Optimization techniques have been made to improve the performance of splitters by developing better junctions and minimizing backward reflections [19-24]. To overcome these difficulties, PCs line defect waveguides and coupling properties should be used. Power splitters based on couplers have previously been reported in the literature [25]. In this paper, we proposed a new mechanism for performing two optical communication wavelengths $1493.6 \mathrm{~nm}$ and $1553 \mathrm{~nm}$.Our suggested design is based on directional couplers and resonant cavities (RC) using $\mathrm{T}$ - branch structure. The coupling region consists of three entire rows of decreased dielectric rods. The plane wave expansion (PWE) [26, $27]$ is employed to calculate photonic band gap (PBG) and demultiplexing function is demonstrated using finite difference time domain (FDTD)[28]. The proposed structure is very compact. And it can be used in the transmission systems and network applications.

\section{Basic photonic crystal design}

The basic proposed structure is formed by square lattice of silicon ( $\mathrm{Si}$ ) rods immersed in air background with refractive index of 3.4, which is showed in Figure 1 The radius of the rods is $r=0.19 a$, where $a$ is the lattice constant. The photonic band gap (PBG) diagram of the basic structure, calculated using the PWE method is depicted in Figure 2. One TE mode which is indicated by blue dashed-line region is presented in photonic crystal structure. As shown in Figure 2, the normalized frequency is observed from $0.2953(a / \lambda)$ to $0.4329(a /$ $\lambda)$, which is sufficiently wide, $\lambda$ is the vacuum wavelength. The frequency lies in the third window of optical communication.

\footnotetext{
* Corresponding author: labbani.amel@umc.edu.dz
} 


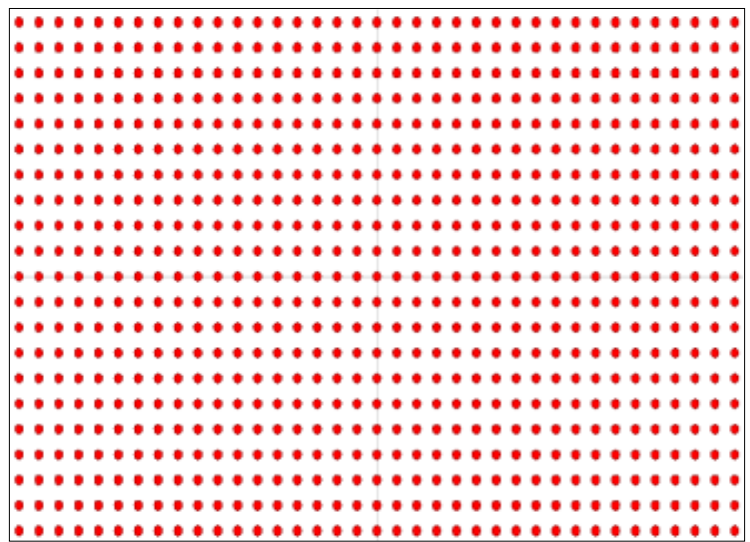

Fig. 1. 2D Photonic crystal structure of square lattice of silicon rods in air background.

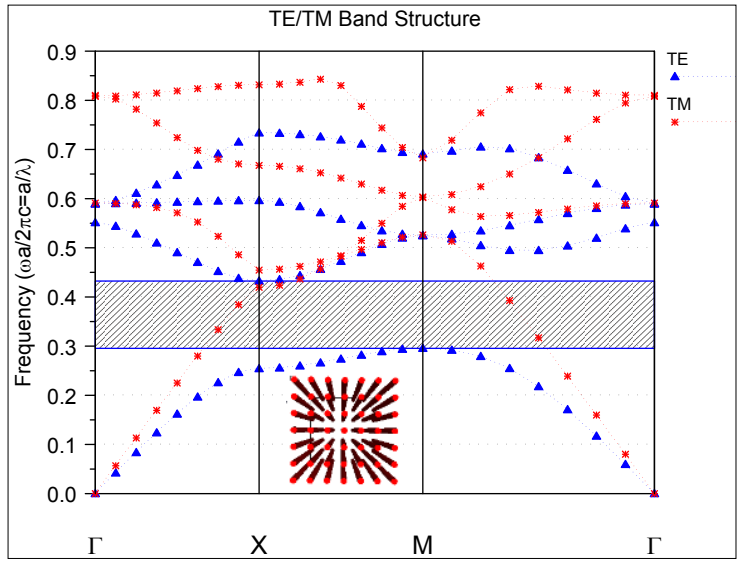

Fig. 2. Band structure of square photonic crystal lattice of Si rods in air matrix for TE polarization

\section{Filter design}

For designing our optical filter, we create two linear waveguides by removing several Si rods in $\mathbf{x}$ direction. They are connected to each other via resonant cavity(RC). RC is obtained by removing a central rod of the basic structure. As shown in Figure 3(a), the radius $R$ of the eight rods situated around the $\mathrm{RC}$ are reduced. A Gaussian signal is applied at the input waveguide, as the incident source, will interact with the resonant cavity and get detected with the monitor placed at the output waveguide. In order to improve the transmission efficiency, two neighbour rods in each side of the cavity are vertically shifted down with a distance $d$ equal to $0.3418 * a$, ( Figure $3(\mathrm{~b})$ ). By choosing different values for $R$ we can obtain different resonant wavelengths. Figure 4 , illustrates that for $\mathrm{R}=0.1 a, 0.1025 a, 0.105 a$, $0.1075 a$ and $0.11 a$, the output spectra of the proposed filter can select different resonant wavelengths of 1501.2 $\mathrm{nm}, 1514 \mathrm{~nm}, 1526.2 \mathrm{~nm}$ and $1540.3 \mathrm{~nm}$, respectively. According to the Figure 4, it has been shown that an increase in $R$ results in red shift in resonance wavelengths because of a decrease in the cavity size. When the light signal is applied to the input waveguide, it is trapped in the resonant cavity and can then be extracted to the output port.

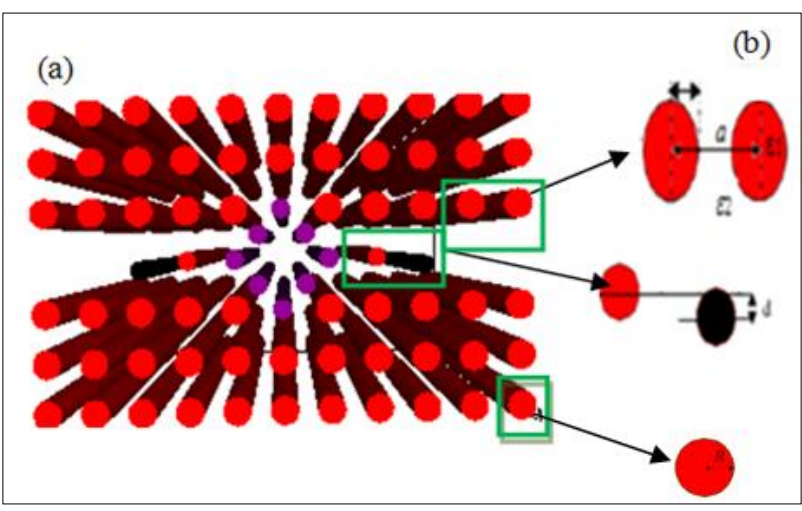

Fig. 3. a) Schematic diagram of the proposed filter, b) a macroscopic view of the resonant cavity.

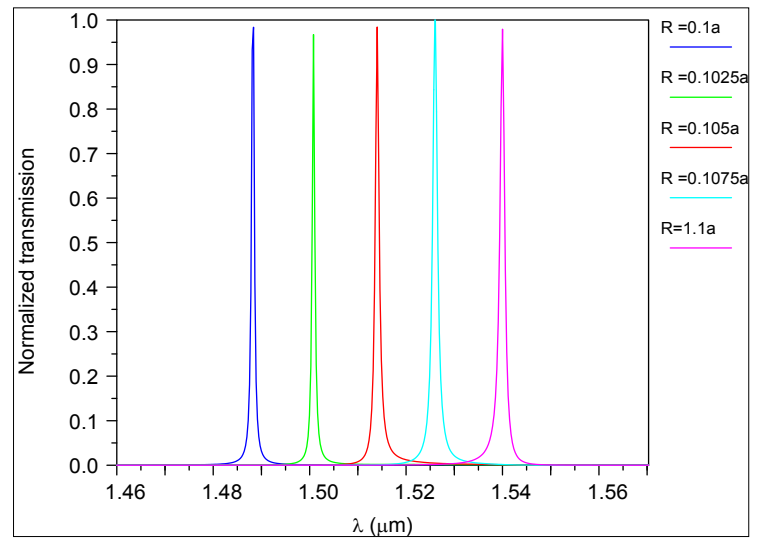

Fig. 4. Schematic spectra of the filter for different values of R.

A crucial parameter in evaluating the performance of the filter is the quality factor $(Q)$ which which is defined as $\lambda 0 / \Delta \lambda$ where $\lambda 0$ is the central wavelength of the transmission and $\Delta \lambda$ is the full width at half maximum of the output intensity.

\section{Directional coupler design}

As displayed in Figure 5, the directional coupler (DC) is formed by creating two straight line defect waveguides separated by three rows of silicon rods whose radius is $r c<r$, where $r$ is the radius of the proposed structure. Decreasing three entire rows of Si rods of the DC create even and odd modes regarding the symmetry plane between the waveguides. These guided modes are known as super-modes. $L c$ is the coupling region between the two waveguides, and defined as the propagation of distance for a total power transferring. The coupling length is important in DC performance and may become significantly small. $L c$ should be integral times of $a$ and can be defined as [8].

$$
L c=2 \pi / \mid k \text { even }-k \text { odd } \mid
$$


$L c$ can be obtained by choosing a normalized frequency $a / \lambda$ on the vertical axis, and by identifying the corresponding phase shift between the two super-modes. In our case the smallest coupling length is about $7 a$.

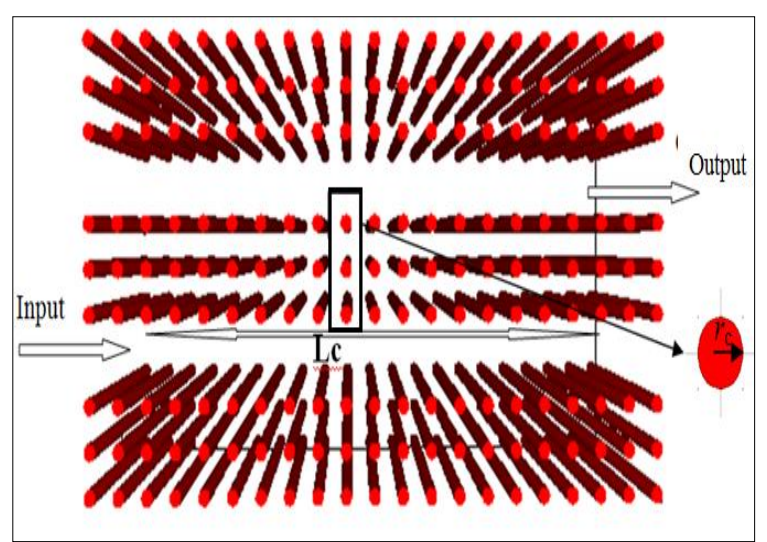

Fig. 5. Schematic diagram of photonic crystal DC which is formed by creating two waveguides separated by three rows of Si rods.

Figure 6 illustrates the band structure of our design, there are two guided modes $k$ (even) and $k$ (odd) inside the band gap.

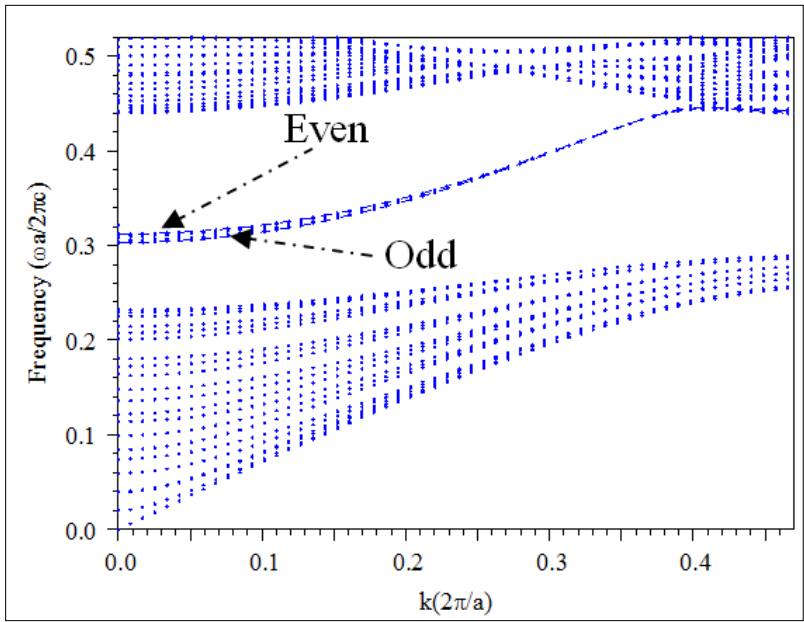

Fig. 6. The super-modes $k$ (even) and $k$ (odd) inside the band gap

By changing $L c$ of the proposed directional coupler, different output power levels can be obtained. Figure 7 shows the relation of the normalized transmission and the coupling length for the two signals. Simulation results using FDTD shows that when $r c=0.111 a$ and $L c=7 a$, the transmission response of $1493.6 \mathrm{~nm}$ and $1553 \mathrm{~nm}$ are more than $90 \%$. Figure 8 also illustrate the change of the radius $r c$ of the three central rows on the output light energy. It has been observed from the Figure 8 that when rc increases the transmission efficiency augments.

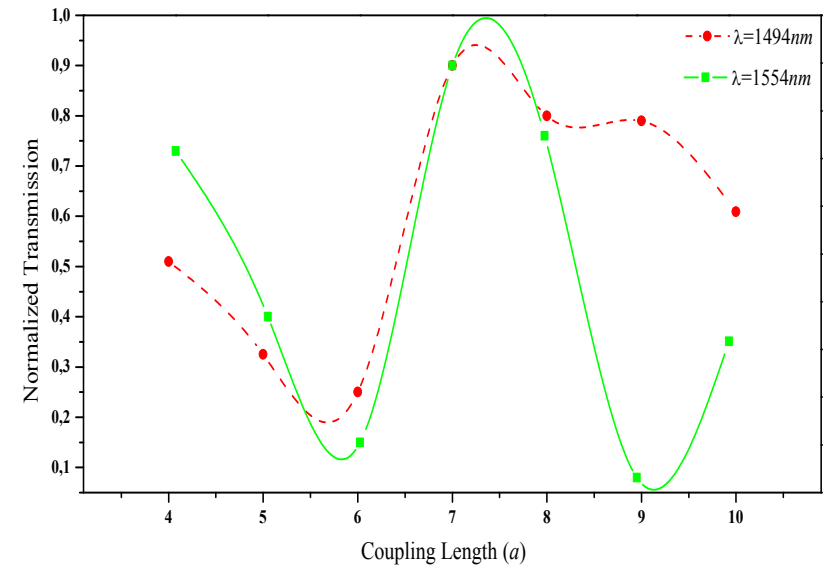

Fig. 7. Output power for wavelengths $1493.6 \mathrm{~nm}$ and $1553 \mathrm{~nm}$ versus coupling lengths.

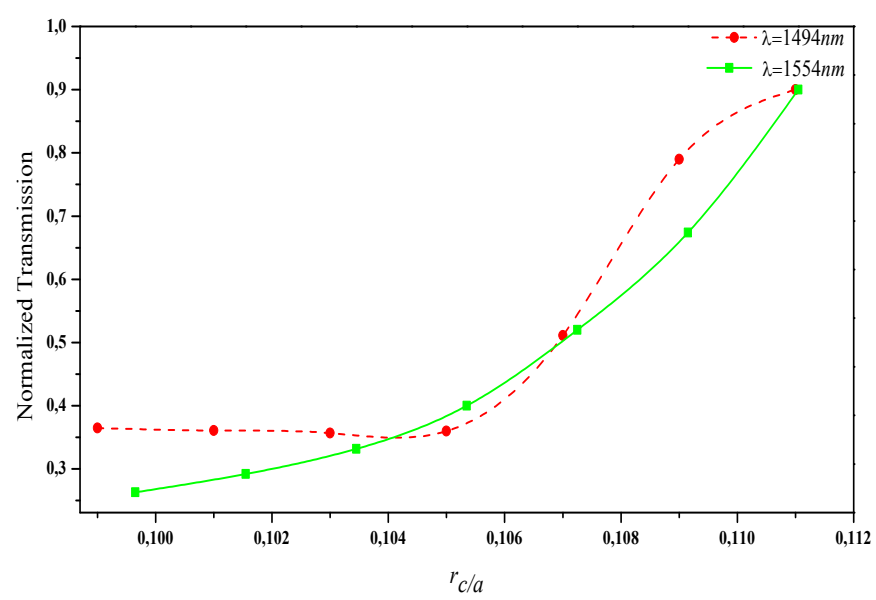

Fig. 8. Output power for wavelengths $1493.6 \mathrm{~nm}$ and $1553 \mathrm{~nm}$ versus radius $r c / a$ of the three central rows.

\section{T- junction diplexer design}

For designing our T- shaped diplexer, we create three linear waveguides, two resonant cavities and two coupling region $(\mathrm{C} 1$ and $\mathrm{C} 2)$. The number of circular rods in $\mathbf{x}$ and $\mathbf{z}$ direction is 70 and 16, respectively. The offprint of the suggested structure is $272.214 \mu \mathrm{m}^{2}$, its dimension is small for integrating. As shown in Figure 9, the two horizontal waveguides contain resonant cavity. $L c$ and $r c$ are $7 a$ and $0.111 a$, respectively these values have given better coupling in the previous section. Fullwave toolbox of RSoft software was utilized to implement FDTD method for simulating the propagation of optical wave in the suggested design. It is used to calculate the transmission structure of our proposed design. Berenger's perfectly matched layer (PML) are applied around the entire T-junction diplexer design as absorbing boundary condition. Figure 10 shows that to achieve two communication wavelengths 1493.6 and $1553 \mathrm{~nm}$, the radius of the cavities $R$ should be equal to $0.08 a$ and $0.11 a$, respectively. 


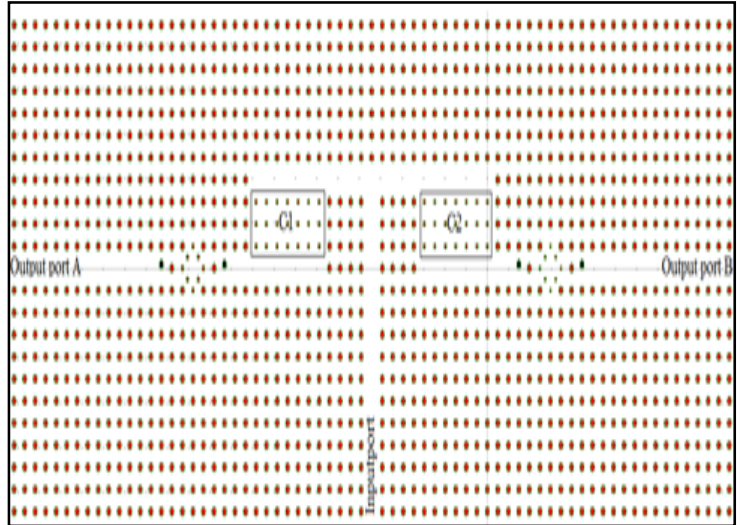

Fig. 9. The schematic structure of the suggested T-shaped diplexer.

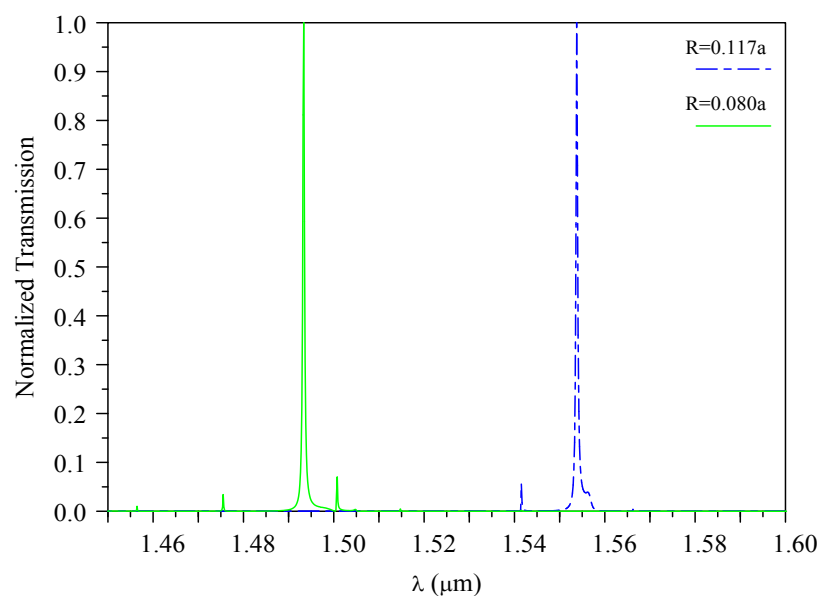

Fig. 10. Output spectra of the proposed T- shaped diplexer for the two wavelengths $1493.6 \mathrm{~nm}$ and $1553 \mathrm{~nm}$.

Table 1. Significant parameters of our T- shaped diplexer.

\begin{tabular}{|c|c|c|c|}
\hline $\begin{array}{c}\text { Wavelength } \\
\text { (nm) }\end{array}$ & $\begin{array}{c}\text { Transmission } \\
\text { efficiency \% }\end{array}$ & $\begin{array}{c}\text { Quality } \\
\text { factor }\end{array}$ & $\begin{array}{c}\text { Extinction } \\
\text { ratio (dB) }\end{array}$ \\
\hline 1493.6 & 99.8 & 2987.2 & -39.7 \\
1553 & 99.7 & 3882.5 & -38.48 \\
\hline
\end{tabular}

As shown in table 1. T-shaped diplexer design has a high transmission efficiency, quality factor and a good crosstalk. In order to confirm the performance of the proposed diplexer, the steady-state electric field distribution for two different wavelengths is depicted in Figure 11. Output power is determined by the time monitors at output end of the two output ports. When the signal launched in the input port, the two wavelengths $1493.6 \mathrm{~nm}$ and $1553 \mathrm{~nm}$ are separated to port B and port A. Hence, the demultiplexing effect is clearly observed.

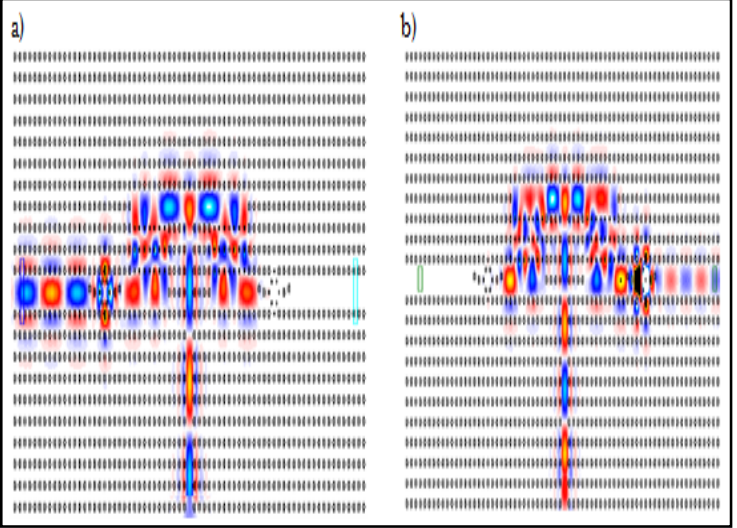

Fig. 11. Optical field distribution for T- shaped diplexer with different wavelengths (a)1553nm, (b)1493.6nm.

\section{Conclusion}

In this letter, a $\mathrm{T}$-shaped optical diplexer in $2 \mathrm{D}$ photonic crystal is investigated. For designing our structure directional couplers between the input and output waveguides are used. The suggested device is used to separate two telecommunication wavelengths. The average output efficiency and quality factors are $99.75 \%$ and 3434.85 respectively. The proposed design has a very low cross talk of -38.48 to $-39.7 \mathrm{~dB}$. The total footprint of our diplexer is $272.214 \mu \mathrm{m}^{2}$ which is very suitable for nanotechnology based demultiplexing applications. Our designed structure has much better performance compared with other works [29-32].

\section{References}

1. E. Yablonovitch, Phys. Rev. Lett. 58, 2059 (1987)

2. S. John, Phys. Rev. Lett. 58, 2486 (1987)

3. J. D. Joannopoulos, R. D. Meade, J. N. Winn, Flow of Light 1 ed. (Princeton University Press, Princeton, 1995)

4. A.E. Akosman, M. Mutlu, H. Kurt, E. Ozbay, Opt. Expr. 19, 24129 (2011)

5. S.B. Haghighi, R. Ghayour, B. Vakili, Optik 124, 6292 (2013)

6. A. Martinez, F. Cuesta, J. Marti, Photonic. Tech. Lett. 15, 694 (2003)

7. K.A. Meradi, F. Tayeboun, S. Ghezali, R. Naoum, H.T. Hattori, Laser Photon. Rev. 32, 572 (2011)

8. M. Koshiba, J. Lightw. Technol. 19, 1970 (2001)

9. R. Talebzadeh, M. Soroosh, T. Daghooghi, IETE Journal Of Research 62, 866 (2016)

10. B. Chen, C. Liu, G. Liu, Opt. Commun. 285, 41 (2012)

11. A. Martinez, J. Bravo-Abad, Fiber and Integrated Optics 22, 151 (2003)

12. S. Bouamami, R. Naoum, Optik 125, 7072 (2014)

13. R. Talebzadeh, M. Soroosh, Y.S. Kavian, F. Mehdizadeh, Optik 140, 331 (2017)

14. H. Alipour-Banaei, M. Soroosh, F. Mehdizadeh, Optik 127, 8706 (2016) 
15. H. AlipourBanaei, F. Mehdizadeh, S. Serajmohammadi, Optik 124, 5964 (2013)

16. M.R. Rakhshani, M.A. Mansouri-Birjandi, Physica E 50, 97 (2013)

17. A. Labbani, A. Benghalia, Chinese Phys. Lett. 32, 054204 (2015)

18. A. Pashaei, A. Andalib, H. AlipourBanaei, J. Telecommun. Devices 3, 35 (2014)

19. C. Chaudhari, D.S. Patil, D.K. Gautam, Opt. Commun. 193, 121 (2001)

20. P.I. Borel, L.H. Frandsen, A. Harpoth, M. Kristensen, J.S. Jensen, O. Sigmund, Electron. Lett. 41, 69 (2005)

21. A. Rostami, H. Alipourbanaei, F. Nazari, A. Bahrami, Optik 122, 1481 (2011)

22. L. Li, G.Q. Liu, H.D. Lu, Optik 124, 2913 (2013)

23. S. Rawal, R.K. Sinha, Opt. Commun. 282, 3889 (2009)

24. F. Monifi, M. Djavid, A. Ghaffari, M.S. Abrishamian, J. Opt. Soc. Am. B 25, 1805 (2008)

25. M. Mohammadi, M.A. Mansouri-Birjandi, IJSTE Transactions Elec. Eng. 39, 93 (2015)

26. S.G. Johnson, J.D. Joannopoulos, Opt. Express 8, $173(2001)$

27. H. A. Haus, Waves and Fields In Optoelectronics (Prentice- Hall, 1984)

28. A. Taflove, S. C. Hegnese, Computational Electrodynamics: The Finite-Difference TimeDomain Method, 3rd ed.( Artech House, Boston, Ma, Usa, 1998)

29. M.R. Rakhshani, M.A. Mansouri-Birjandi, IJCSI 9, 24 (2012)

30. S. Naghizade, S.M. Sattari-Esfahlan, Photon. Netw. Commun. 34, 445 (2017)

31. Y. D. Wu, T.T. Shih, J.J. Lee, 2009 Asia Communications and Photonics Conference and Exhibition (ACP)Shanghai, China, 2-6Nov. IEEE, (2009)

32. T.T. Shih, Y.D. Wu, J.J. Lee, Photonic. Tech. Lett. 21, 18 (2009) 